\title{
Microimaging FT-IR of Head and Neck Tumours. The case of salivary glands \\ Carla Conti $^{1}$, Paolo Ferraris ${ }^{1}$, Elisabetta Giorgini ${ }^{1}$, Corrado Rubini ${ }^{2}$, Simona Sabbatini ${ }^{1}$ and Giorgio Tosi*1
}

\author{
Address: ${ }^{1}$ Dipartimento ISAC sez. Chimica, Università Politecnica delle Marche, Ancona, Italy and ${ }^{2}$ Dipartimento di Neuroscienze, Università \\ Politecnica delle Marche, Ancona, Italy \\ * Corresponding author
}

\author{
from Ist Scientific Meeting of the Head and Neck Optical Diagnostics Society \\ London, UK. 14 March 2009 \\ Published: 28 July 2009 \\ Head \& Neck Oncology 2009, I(Suppl I):OI3 doi:I0.II86/I758-3284-I-SI-OI3
}

This abstract is available from: http://www.headandneckoncology.org/content/I/SI/OI3

(c) 2009 Conti et al; licensee BioMed Central Ltd.

The potential role of IR spectroscopy in biomedical science has been exploited to distinguish different biomolecules by probing chemical bond vibrations and using these molecular and sub-molecular patterns to define and differentiate pathological from healthy samples.

This technique aims to further exploit the potentiality of infrared spectroscopy in isolating and defining spectral profiles in salivary glands attributable to various kinds of cancer: Warthin tumour, polymorphous low-grade adenocarcinoma, oral epithelium with dysplasia, adenoid cystic carcinoma, lymphoma, and the corresponding healthy tissues. Thirty three samples from patients with diagnosed salivary gland pathology were analyzed. Two adjacent sections of tissues ( $5 \mu \mathrm{m}$ thickness) were used for both histopathological and FTIR analysis.

Spectral data were achieved with Perkin Elmer (PE) Spectrum One FT-IR and Spotlight FT-IR Imaging System 400 spectrometers. Data handling: PE Spectrum v.6.3.1, Grams AI (Galactic) and Pirouette 4.0 (Imfometrix), PE Hyper View Images software. Changes were monitored at the molecular level, probing spectral markers such as Amide I and II, phosphate, nucleic acids, and carbohydrates vibrational modes.

In order to further verify the reliability of our classification, all the spectra from cancerous regions were mixed with those from zones characterized by other tumours obtaining a HCA grouping in excellent agreement with their biochemical and histological characterization.

The results once again demonstrates the capability of infrared microspectroscopy imaging, in combination with multivariate data analysis, to highlight even subtle biochemical and morphological changes, distinguishing various kinds and grades of neoplasia in human tissues. The rapid images acquisition at a high-spatial resolution can be fully satisfied with the use of multi detectors Spotlight system or synchrotron light sources operating at and down the diffraction limit. On considering this and our previous reports, the potentiality of spectral analysis in the study of head and neck neoplasia can be outlined. The complexity of human tissues requires more experimental efforts and statistical evidence to unambiguously formulate a diagnosis of a pathology at a molecular level, mainly at an early stage, this technique has been a valid and synergistic support to the classical histopathological screening. 